\title{
Modified Moments and Maximum Likelihood Estimators for Parameters of Erlang Truncated Exponential Distribution
}

\author{
Kannadasan Karuppaiah, Vinoth Raman
}

\begin{abstract}
This study derives the parameter estimation in truncated form of a continuous distribution which is comparable to Erlang truncated exponential distribution. The shape and scale parameter will predict the whole distributions properties. Approximation will be useful in making the mathematical calculation an easy understand for non-mathematician or statistician. An explicit mathematical derivation is seen for some properties of, Method of Moments, Skewness, Kurtosis, Mean and Variance, Maximum Likelihood Function and Reliability Analysis. We compared ratio and regression estimators empirically based on bias and coefficient of variation.

Keywords: Erlang-Truncated Exponential Distribution, Maximum Likelihood Function, Moments, Moment Generating Function, Reliability Analysis.
\end{abstract}

$$
f(x)_{E T E D}=\theta\left(1-e^{-\lambda}\right) e^{\theta x\left(1-e^{-\lambda}\right)} \quad x \geq 0, \theta, \lambda>0
$$

The corresponding the Probability distribution function is

$$
F(x)_{E T E D}=1-e^{-\theta x\left(1-e^{-\lambda}\right)}
$$

And corresponding Survival Function (S.F) is

$$
\bar{H}(x)_{E T E D}=e^{-\theta x\left(1-e^{-\lambda}\right)}
$$

ETED is commonly seen in the area of queuing and stochastic processes. This family of distribution derived from the exponential distribution. More details study can be seen in (Ei-Alosey, 2017) who initially introduced this (ETED), (Ahsanullah, M 1991 and Ahsanullah, M 1992) has estimated the distributional properties of the parameter archives through Lomax Distribution.

Manuscript received on 04 April 2021 | Revised Manuscript received on 08 April 2021 | Manuscript Accepted on 15 April 2021 | Manuscript published on 30 April 2021.

* Correspondence Author

Kannadasan Karuppaiah*, Assistant Professor, Department of Community Medicine, Melmaruvathur Adhiparasakthi Institute of Medical Sciences and Research, Melmaruvathur - $\quad 603 \quad 319 . \quad$ Tamilnadu, India.statkannadasan@gmail.com

Vinoth Raman, Assistant Professor, Department of Quality Measurement and Evaluation, Deanship of Quality and Academic Accreditation, Imam Abdulrahman Bin Faisal University, P. O. Box 1982, Dammam - 31441. Saudi Arabia.

(C) The Authors. Published by Lattice Science Publication (LSP). This is an open access article under the CC-BY-NC-ND license (http://creativecommons.org/licenses/by-nc-nd/4.0/)

\section{INTRODUCTION}

A Random Variable (r.v) $X$ following Erlang Truncated Exponential Distribution (ETED), if its density function (p.d.f) is given by

(Balakrishnan, 1994) derived the repetition relations for moments estimation of record values through Gumbel distribution. (Ahsanullah.M, 1995) derived some moments of the distribution properties in each records.In this Study we have derived and estimated the MLE on Erlang Truncated Exponential model and discussed some of its statistical properties. This study is structured as follows: Moment Generating function is given in section 2.1, Method of Moments, Skewness, Kurtosis, Mean and Variance has been given in section 2.2, Maximum Likelihood Function in section 2.3 and Reliability analysis is specified in section 2.4.The inference in section 3 . 


\section{MATHEMATICAL FORMULATION}

2.1 Moment Generating Function (M.G.F)

This sub-section derived the (m.g.f) of ETED

$$
\begin{aligned}
& E\left(e^{t x}\right)=\int_{0}^{\infty} e^{t x} f(x) d x \\
= & \int_{0}^{\infty} e^{t x} \theta\left(1-e^{-\lambda}\right) e^{\theta x\left(1-e^{-\lambda}\right) d x} \\
= & \theta\left(1-e^{-\lambda}\right) \int_{0}^{\infty} e^{t x-\theta x\left(1-e^{-\lambda}\right)} d x \\
= & \frac{\theta\left(1-e^{-\lambda}\right)}{\theta\left(1-e^{-\lambda}\right)-t} \\
= & {\left[1-\frac{t}{\theta\left(1-e^{-\lambda}\right)}\right]^{-1} }
\end{aligned}
$$

\subsection{Method of Moment Estimation}

The r.v derived in moments $\bar{X}=$ indicated in (5). The likelihood corresponding to the sample $X_{1}, X_{2}, X_{3}, \ldots X_{n}$ as a random sample on $X$ having p.d.f

$$
\begin{aligned}
& E^{r}=1+\frac{t}{\theta\left(1-e^{-\lambda}\right)}+\frac{t^{2}}{\theta^{2}\left(1-e^{-\lambda}\right)^{2}}+\cdots \\
& \mu_{1}^{\prime}=\frac{1}{\theta\left(1-e^{-\lambda}\right)} \\
& \mu_{2}^{\prime}=\frac{2}{\theta^{2}\left(1-e^{-\lambda}\right)^{2}} \\
& \mu_{3}^{\prime}=\frac{6}{\theta^{3}\left(1-e^{-\lambda}\right)^{3}} \\
& \mu_{4}^{\prime}=\frac{24}{\theta^{4}\left(1-e^{-\lambda}\right)^{4}}
\end{aligned}
$$

Based on the equation (6), coefficient of Skewness, Kurtosis and Mean, Variance; ETED been attained through the following relation

$$
\begin{aligned}
\operatorname{SKE}_{\mathrm{ETED}} & =\frac{4 / \theta^{6}\left(1-\mathrm{e}^{-\lambda}\right)^{6}}{1 / \theta^{6}\left(1-\mathrm{e}^{-\lambda}\right)^{6}} \\
\operatorname{KUR}_{\text {ETED }} & =\frac{9 / \theta^{4}\left(1-\mathrm{e}^{-\lambda}\right)^{4}}{1 / \theta^{4}\left(1-\mathrm{e}^{-\lambda}\right)^{4}}
\end{aligned}
$$

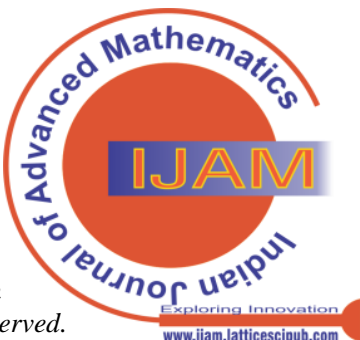




$$
\begin{aligned}
& \mathrm{E}(\mathrm{X})_{\mathrm{ETED}}=\frac{1}{\theta\left(1-\mathrm{e}^{-\lambda}\right)} \\
& \mathrm{V}(\mathrm{X})_{\mathrm{ETED}}=\frac{1}{\theta^{2}\left(1-\mathrm{e}^{-\lambda}\right)^{2}}
\end{aligned}
$$

\subsection{Maximum Likelihood Function}

The R.V derived through likelihood; $\bar{X}=$ indicated in (1). The likelihood corresponding to the sample $X_{1}, X_{2}, X_{3}, \ldots X_{n}$ as a random sample on $X$ having p.d.f is

$$
L=\prod_{i=1}^{n} \theta\left(1-e^{-\lambda}\right) e^{\theta x\left(1-e^{-\lambda}\right)}
$$

$$
=\theta^{n}\left(1-e^{-\lambda}\right)^{n} e^{-\theta\left(1-e^{-\lambda}\right) \sum_{i=1}^{n} x}
$$

The log likelihood becomes

$\log L=\log \theta^{n}+\log \left(1-e^{-\lambda}\right)^{n}-n \theta \mu\left(1-e^{-\lambda}\right)$

Maximum Likelihood Estimator (MLE) is derived as

$\frac{\partial \log L(\theta / x)}{\partial \theta}=0$

ML Estimator $\theta$ is solution of

$$
\frac{\partial \log L}{\partial \theta}=\frac{n}{\theta}-n \mu\left(1-e^{-\lambda}\right)
$$

ML Estimator $\lambda$ is solution of

$$
\frac{\partial \log L}{\partial \lambda}=\frac{n \lambda e^{-\lambda}}{\left(1-e^{-\lambda}\right)}-n \theta \mu \lambda e^{-\lambda}
$$

ML Estimator $\mu$ is solution of

$$
\frac{\partial \log \mathrm{L}}{\partial \mu}=\mathrm{n} \theta\left(1-\mathrm{e}^{-\lambda}\right)
$$

MLE, of $\theta, \lambda$ and $\mu$ is attained through the nonlinear classification of equations. The location of these parameters to zero and solving simultaneously yields the MLE of three parameters. To obtain, elements of information matrix, we will get the second order derivative of logarithms of the $\frac{\partial^{2} \log L}{\partial \theta^{2}}=-\frac{n}{\theta^{2}}$

$$
\begin{aligned}
\frac{\partial^{2} \log L}{\partial \lambda^{2}} & =\frac{\left(1-e^{-\lambda}\right)\left(e^{-\lambda}+\lambda e^{-\lambda}(-\lambda)\right)-\lambda^{2} e^{-\lambda}\left(e^{-\lambda}\right)}{\left(1-e^{-\lambda}\right)^{2}} \\
& =\frac{e^{-\lambda}-\lambda^{2} e^{-\lambda}-e^{-2 \lambda}}{\left(1-e^{-\lambda}\right)^{2}} \\
& =\frac{e^{-\lambda}\left(1-\lambda^{2}-e^{-\lambda}\right)}{\left(1-e^{-\lambda}\right)^{2}}
\end{aligned}
$$

$\frac{\partial^{2} \log L}{\partial \mu^{2}}=0, \quad \frac{\partial^{2} \log L}{\partial \theta \partial \mu}=-n\left(1-e^{-\lambda}\right), \quad \frac{\partial^{2} \log L}{\partial \theta \partial \lambda}=-n \mu \lambda e^{-\lambda}, \quad \frac{\partial^{2} \log L}{\partial \lambda \partial \mu}=-n \theta \lambda e^{-\lambda}$ $\frac{\partial^{2} \log L}{\partial \lambda \partial \theta}=-n \mu \lambda e^{-\lambda}, \quad \frac{\partial^{2} \log L}{\partial \mu \partial \theta}=n\left(1-e^{-\lambda}\right), \quad \frac{\partial^{2} \log L}{\partial \mu \partial \lambda}=n \theta \lambda e^{-\lambda}$ 
For interval estimation of $(\theta, \lambda, \mu)$ the observed information matrix is obtained since, its probability involves mathematical combination. The $3 \times 3$, observed information matrix $J(\theta)$ is

$J(\theta)=-\left(\begin{array}{ccc}I_{\theta \theta} & I_{\theta \lambda} & I_{\theta \mu} \\ I_{\lambda \theta} & I_{\lambda \lambda} & I_{\lambda \mu} \\ I_{\mu \theta} & I_{\mu \lambda} & I_{\mu \mu}\end{array}\right)$

Whose elements are given above, under regularity conditions, the asymptotic distribution of

$$
\sqrt{n}(\hat{\theta}-\theta) \text { is } N_{3}\left(0, I \theta^{-1}\right)
$$

Where, $I(\theta)$ is the estimated information matrix. This asymptotic distribution performance is effective if $I(\theta)$ is substituted by $J(\theta)$, i.e., the observed information matrix assessed at $\hat{\theta}$.

\subsection{Reliability Analysis}

The S.F, also identified as reliability function in engineering, is the representative of an explanatory variable that plots a set of measures, generally related with failure of some structure onto time. We assume, the probability that the structure will survive beyond an indicated time.

Reliability function $R(t)$, is the chance of an item not failing prior to a one-time $t$, which is defined by; $R(t)=1-F(t)$. The $R(t)$ of an ETED is assumed as

$$
R(t)=1-e^{-\theta x\left(1-e^{-\lambda}\right)}
$$

Other parameterconcern of an r.v is the hazard rate function assumed by

$h(t)=\frac{f(t)}{1-F(t)}$

This is an important quantity characterizing life phenomenon. It can be loosely interpreted as the conditional probability of failure, given it has survived to the time $t$. The hazard rate function for an ETED, r.v is given by

$h(t)=\frac{\theta\left(1-e^{-\lambda}\right) e^{\theta x\left(1-e^{-\lambda}\right)}}{1-e^{-\theta x\left(1-e^{-\lambda}\right)}}$

\section{CONCLUSION}

We derived some expression and recurrence for moments and M.G.F to observe, values of ETED. Further, the estimation of parameters is derived by method of ML and obtained the information matrix.

\section{REFERENCE}

1. A.A. Jamjoom and Z.A. AL-Saiary. (2010). "Moments of Order Statistics from Non-identically Distributed Three Parameters Beta type-I and Erlang Truncated Exponential Variables", Journal of Mathematics and Statistics, Vol, 6(4), pp.442-448. [CrossRef]

2. A.R. El-Alosey, (2007), "Random sum of new type of mixture of distribution", International. Journal of Statistical and System, Vol, 2, pp.49-57.
3. Anamika Kulshrestha, R. U. Khan, Devendra Kumar. (2012), “On Moment Generating Function of Generalized Order Statistics from Erlang-Truncated Exponential Distribution", Open Journal of Statistics, Vol, 2, pp.557-564. [CrossRef]

4. Imtiyaz a. Shah, (2013). "On a characterization of generalized Erlang-truncated exponential distribution through distributional properties of dual generalized order statistics", Tamkang journal of mathematics, Vol.44, No. 4, pp.445-452. [CrossRef]

5. Muhammad Mohsin, (2009), "Recurrence Relation for Single and Product Moments of Record Values from Erlang-truncated Exponential Distribution", World Applied Sciences Journal, Vol.6 (2), pp. 279-282.

6. Ahsanullah, M., (1991). "Record values of Lomax distribution", Statisti. Nederlandica, Vol.41, No.1, pp. 21-29. [CrossRef]

7. Ahsanullah, M., (1992). "Record values of independent and identically distributed continuous random variables", Pak. J. Statist., Vol.8, No.2. Pp. 9-34.

8. Ahsanullah, M., (1995). "Record Statistics", Nova Science Publishers, USA.

9. Balakrishnan, N. and M. Ahsanullah, (1994). "Recurrence Relation for single and product moments of record values from exponential generalized Pareto distribution". Comm. Stat. Methods, Vol.23, No.10, pp.2841-2852. [CrossRef]

10. Balakrishnan, N. and M. Ahsanullah, (1994). "Recurrence Relations for single and product moments of record values from Lomax distribution”, Sankhya, Vol.56, B2, pp.140-146.

\section{AUTHORS PROFILE}

Dr. K.Kannadasan, Assistant Professor from Melmaruvathur Adhiparasakthi Institute of Medical Sciences and research, Melmaruvathur. Skilled in Bio-Statistics, Statistical software, Stochastic

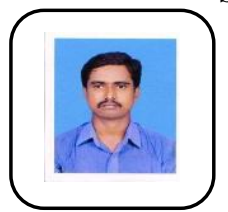
Modelling and survival analysis. Strong educational professional with sound knowledge in research have a doctor of philosophy (Ph.D.). Having five years medical college field teaching experience, in the department of community medicine department. Published in more than 20 articles and 1 book.

Dr. R.Vinoth, Assistant Professor from Imam Abdul Rahman Bin Faisal University, Kingdom of Saudi Arabia: with a demonstrated history of working in the higher education industry. Skilled in Bio-Statistics,

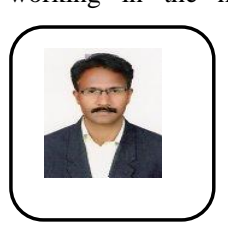
Statistical software, Stochastic Modelling and surviva analysis. Strong educational professional with sound knowledge in research have a doctor of philosophy (Ph.D.). Having two years medical college field teaching experience, in the department of community medicine department. Online teaching experience from Texila American University, as a subject matter expert for the past three years. Published in more than 50 articles and 4 books. 